\title{
Digitale Edition und Harmonische Analyse mit MEI von Anton Bruckners Studienbuch
}

\author{
Agnes Seipelt, Detmold
}

DOI: $10.25366 / 2020.100$

Zitation: Agnes Seipelt, „Digitale Edition und Harmonische Analyse mit MEI von Anton Bruckners Studienbuch”, in: Brückenschläge zwischen Musikwissenschaft und Informatik. Theoretische und praktische Aspekte der Kooperation, in Verbindung mit der Fachgruppe Digitale Musikwissenschaft hrsg. von Stefanie Acquavella-Rauch, Andreas Münzmay und Joachim Veit (= Musikwissenschaft: Aktuelle Perspektiven. Bericht über die Jahrestagung der Gesellschaft für Musikforschung 2019 in Paderborn und Detmold, Bd. 3), Detmold, Musikwissenschaftliches Seminar der Universität Paderborn und der Hochschule für Musik Detmold, 2020, S. 105-113, DOI: 10.25366/2020.100






\title{
Digitale Edition und Harmonische Analyse mit MEl von Anton Bruckners Studienbuch
}

\author{
AGNES SEIPELT, DETMOLD
}

Das Poster mit dem Titel „Digitale Edition und Harmonische Analyse mit MEI von Anton Bruckners Studienbuch" ist eine Zusammenfassung der Ergebnisse, die in dem von 2017 bis 2019 laufenden Projekt „Digitale Musikanalyse mit den Techniken der Music Encoding Initiative (MEI) am Beispiel der Kompositionsstudien Anton Bruckners" erarbeitet wurden. Das von GO!DIGITAL 2.0 geförderte Projekt war ein Kooperationsprojekt der Österreichischen Akademie der Wissenschaften mit dem Zentrum Musik | Edition | Medien und stand unter der Leitung von Robert Klugseder. Mitarbeiter auf österreichischer Seite waren Paul Gulewycz und Peter Provaznik. Zudem standen Johannes Kepper (Musikwissenschaftliches Seminar Detmold/Paderborn) und Laurent Pugin (RISM Schweiz) beratend zur Seite.

Das Projekt hatte einerseits zum Ziel, eine Digitale Edition bzw. Transkription des Studienbuchs von Anton Bruckner vorzulegen, das er während seiner Lehrzeit bei Otto Kitzler von 1861 bis 1863 anfertigte und 2014 in einer Faksimile Ausgabe veröffentlicht wurde. ${ }^{1}$ Andererseits sollte auf Grundlage dieses Notenmaterials eine automatisierte Harmonische Analyse konzipiert werden.

Das Studienbuch umfasst 326 Seiten, auf denen der Kompositionsunterricht Bruckners nachvollziehbar abgebildet ist. Es beginnt mit einfachen Übungen zu Schlussbildungen, Modulationen, später kommen verschiedene Übungen zu musikalischen Formen hinzu und seine sogenannte "Studiensymphonie" schließt das Buch ab. Die (digitale) Transkription der Inhalte erfolgte mit der Auszeichnungssprache $\mathrm{MEI}_{1}{ }^{2}$ einer auf $\mathrm{XML}$ basierten, maschinenlesbaren Sprache, mit der notierten Musik so formalisiert beschrieben werden kann, dass damit Algorithmen ausgeführt und Analysen betrieben werden können. In dem Autograph sind zahlreiche Eingriffe in den Textfluss wie Streichungen, Rasuren, Überschreibungen und Ergänzungen vorgenommen worden, die Variantenbildungen und vor allem Korrekturmaßnahmen zeigen. Hinzu kommen zahlreiche verbale Anmerkungen, die das Notierte kommentieren und ergänzen. Wir gehen davon aus, dass diese Eingriffe hauptsächlich von Bruckners Hand oder von Otto Kitzler stammen. Die genannten Phänomene, die sich in herkömmlichen Editionen im Kritischen Apparat befinden, können mit MEI direkt im Notentext hinterlegt werden, indem sie als Variante bzw. als Streichung und Hinzufügung beschrieben werden. Die MEI-Codierungen

1 Das "Kitzler-Studienbuch": Anton Bruckners Studien in Harmonie- und Instrumentationslehre bei Otto Kitzler (1861-1863). Faksimile-Ausgabe, hrsg. von Paul Hawkshaw und Erich Wolfgang Partsch, Wien 2014.

2 Music Encoding Initiative, <https://music-encoding.org> (10.06.2020). 
sind somit mit allen Informationen versehen, die das Autograph aufzuweisen hat (s. Abb. 1). Ein Rendering der codierten Noten wurde mit der Anzeigebibliothek Verovio ${ }^{3}$ erreicht und diese in die Anwendung des Studienbuch-Viewers ${ }^{4}$ eingebettet. Die genannten Texteingriffe wie Streichungen und Hinzufügungen sind unterschiedlich eingefärbt, wobei die vermutlich früheste Textschicht, die Hinzufügungen, als blau angezeigt werden.

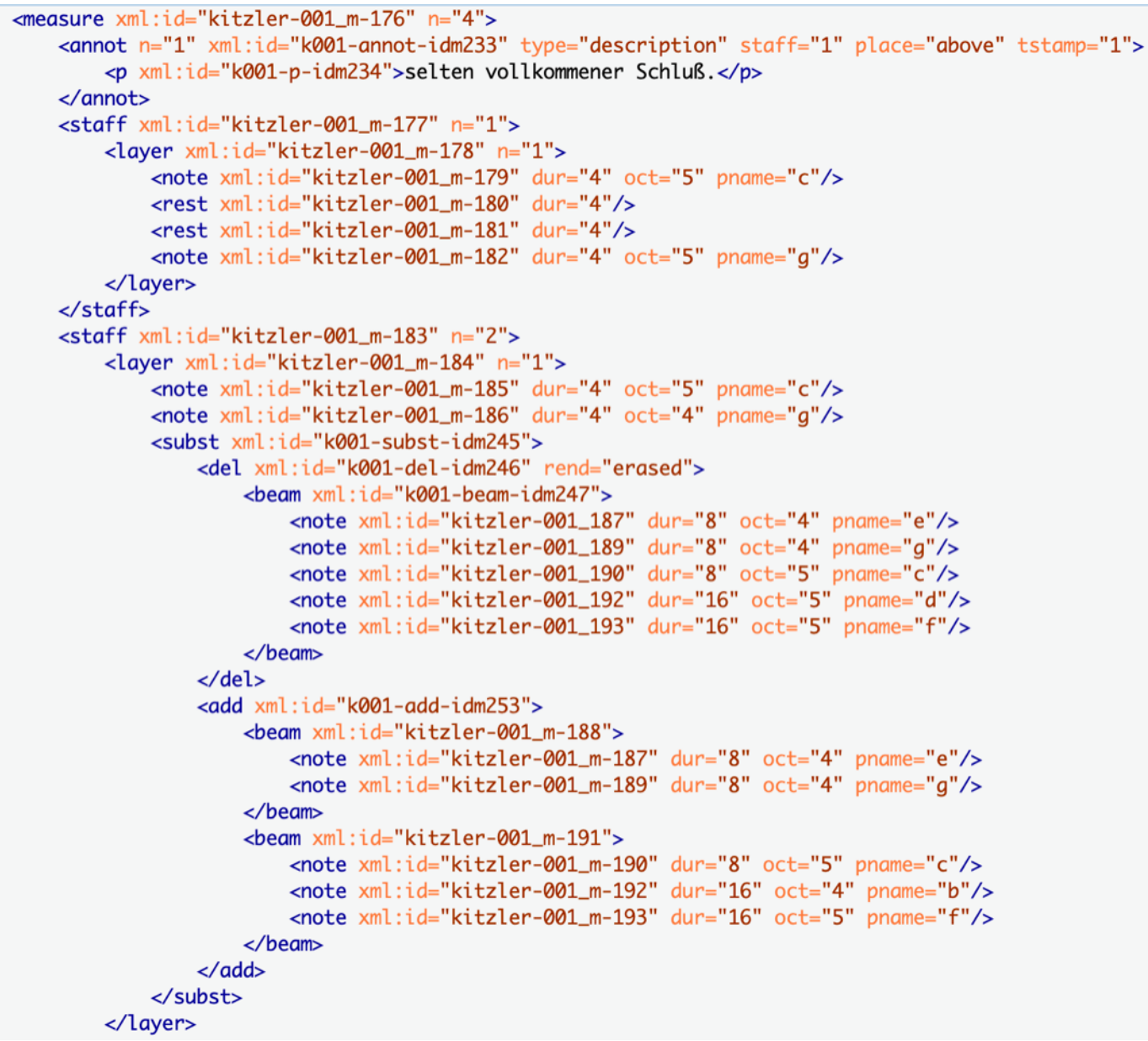

Abbildung 1

Im Studienbuch-Viewer können die Nutzer*innen in einem drop-down Menü zwischen den Ansichten „Edition" und „Analyse" auswählen und das jeweilige Faksimile kann hinzugeschaltet werden. Die Übertragung in der Editionsansicht ist vorwiegend diplomatisch ausgerichtet, $d . h$. dass die Seitenaufteilung an die Vorlage angepasst ist, Zeilenumbrüche wurden übernommen und das Blättern geschieht synchron (s. Abb. 2). Wiederholungsanweisungen von Bruckner wie z. B. ein einfaches "etc", das darauf verweist, dass die letzten Takte genau gleich sind wie in der Übung darüber, werden nicht ausgeführt, um die Topographie des Originals zu erhalten.

3 Verovio. A music notation engraving library, <https://www.verovio.org> (10.06.2020).

4 <https://apps-mufo.oeaw.ac.at/studienbuch/> (12.06.2020). 




Abbildung 2

Die auf den in MEI codierten Daten aufbauende, automatische Harmonieanalyse besteht aus drei Schritten: Tonartenerkennung, Akkorderkennung und Stufenanalyse. Für die Tonartenerkennung wurde ein in der Music Information Retrieval (MIR) bekannter und in anderen Programmen wie Humdrum ${ }^{5}$ oder music $21^{6}$ verwendeter Algorithmus zur automatischen Erkennung von Tonarten verwendet. Dieser Krumhansl-Schmuckler-Algorithmus ${ }^{7}$ basiert auf einem Zusammenzählen der unterschiedlichen klingenden Töne in einem bestimmten Abschnitt, wobei der Algorithmus mit MIDI-Werten arbeitet und somit Oktavlage und Enharmonik für die Berechnungen irrelevant sind. Der Algorithmus besteht im Prinzip aus einer mathematisch trivialen Korrelationsrechnung, mit der die Ähnlichkeit von zwei Werten berechnet werden kann. Mit dem o. g. Auszählen der Tonhöhen von z. B. einem 30-taktigem Lied wird der Input-Vektor erstellt. Dieser enthält für alle zwölf Töne die Angabe, wie oft sie, berechnet auf Vierteln, vorhanden sind. Dieser Vektor $x$ wird mit Referenzwerten (y) abgeglichen, die aus Korpora oder mit Hörexperimenten erstellt wurden. Für jede Tonart gibt es eine Verteilung der Noten nach ihrer Wichtigkeit bzw. Häufigkeit in der betreffenden Tonart. Der Anteil der Töne $c$ und $g$ zum Beispiel ist in C-Dur höher oder sie werden als wichtiger wahrgenommen als beispielsweise der Ton fis bzw. ges. Der Input-Vektor wird mit den Referenzwerten aller Tonarten verglichen. Der daraus resultierende höchste Index (R) gehört demnach zum Referenzwert, der dem Input-Vektor am ähnlichsten ist und dieser wird somit als die wahrscheinlichste Tonart für diesen Abschnitt angesehen. Die Auswahl der zu berechnenden Abschnitte eines Musikstücks kann taktweise eingestellt werden. Diese werden dabei überlappend berechnet, wenn also die Fensterbreite

5 <https://www.humdrum.org/> (14.06.2020).

6 music21: a toolkit for computer-aided musicology, <http://web.mit.edu/music21/> und <https://web.mit.edu/ music21/doc/moduleReference/moduleAnalysisDiscrete.html> (14.06.2020).

7 Carol L. Krumhansl, Cognitive Foundations of Musical Pitch, Oxford 1990, S. 77-110 und Humdrum extras. Keycor manpage, <http://extras.humdrum.org/man/keycor/> (10.06.2020). 
4 gewählt ist, werden zunächst die Takte 1 bis 4 berechnet, dann die Takte 2 bis 5, dann 3 bis 6 usw. Dadurch steht ein Takt ggf. in mehreren Tonarten, je nach dem, in welchem Kontext er sich befindet. So können aber andererseits Tonartenwechsel und sogar Modulationen sichtbar werden. Die Tonarten werden im Studienbuch-Viewer durch verschiedenfarbige Balken unter den Systemen dargestellt, wodurch die Tonartänderung durch Überlappungen der Balken sehr intuitiv sichtbar gemacht wird.

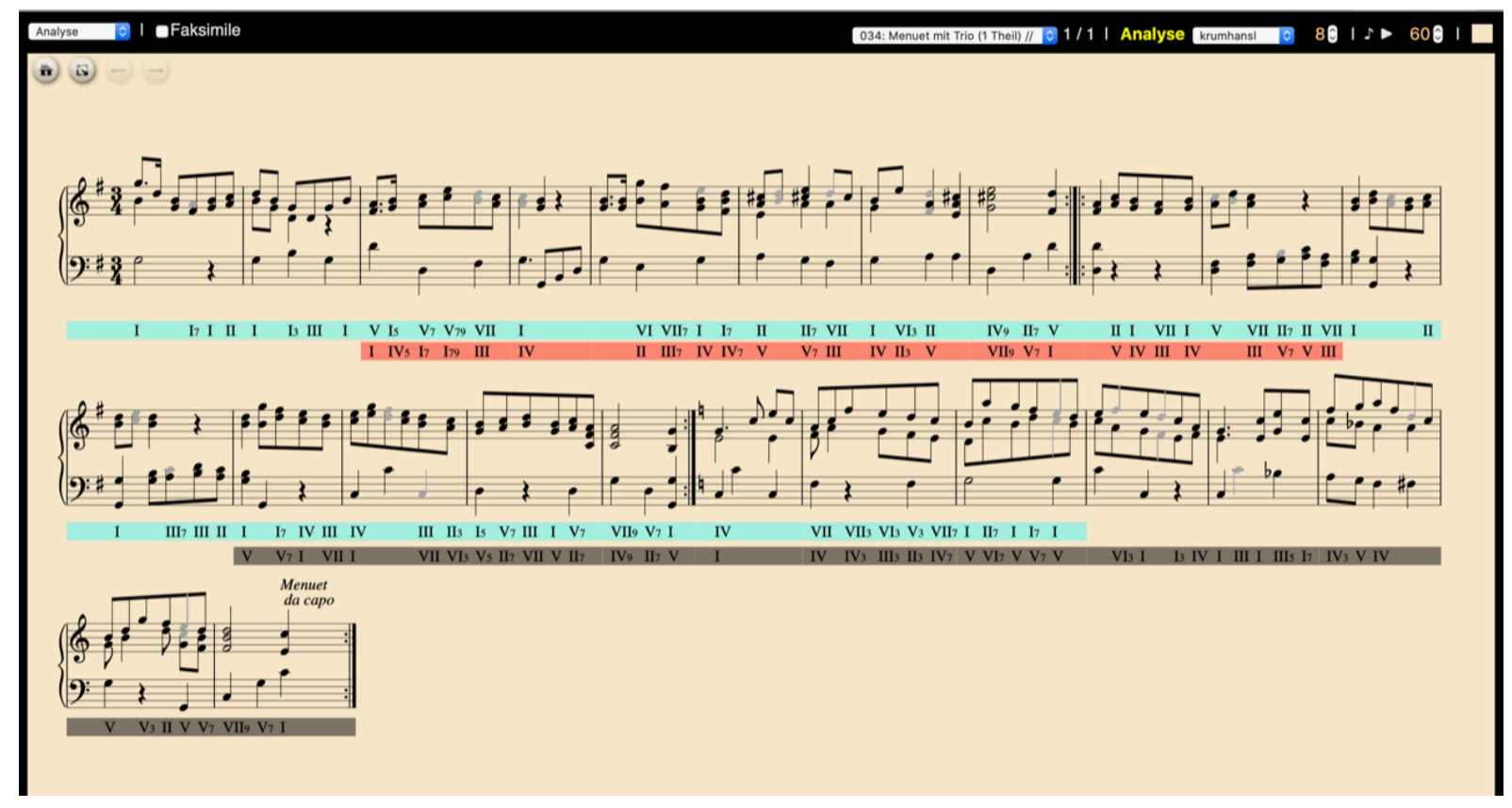

Abbildung 3

In einem zweiten Schritt wird unabhängig von den ermittelten Tonarten eine Akkorderkennung durchgeführt. Technisch basiert diese auf mehreren XSLT ${ }^{8}$-Skripten, einer Programmiersprache, die XML-Daten (also auch MEI-Daten) auswerten, prozessieren und verändern kann. Konzeptionell werden dafür alle zu einer Zählzeit gleichzeitig erklingenden Töne (wenn es mindestens zwei unterschiedliche Tonnamen sind) untersucht und zunächst der Grundton dieses Akkords ermittelt. Dies geschieht über Terzschichtung, wobei davon ausgegangen wird, dass die dichteste Schichtung (= die engste Lage) für den am meisten wahrscheinlichen Akkord steht. Dafür werden die Terzen über dem Grundton durchnummeriert, beispielsweise bekommt die erste Terz über einem c, also e oder es, die Nummer 1, das $g$ (bzw. ges oder gis) die Nummer 2 usw. Dann werden über jeden klingenden Ton aus dem Tonvorrat der gleichzeitig klingenden Tönen Terzen geschichtet, was bedeutet, dass jeder Ton zunächst als Grundton interpretiert wird. Die niedrigste Zahl aller Terzen mit der jeweils höchsten Nummer (= die dichteste Schachtelung) bestimmt dann, über welchem Ton der Akkord wahrscheinlich aufgebaut ist. Beispiel: Aus den

8 Extensible Stylesheet Language Transformation, siehe auch <https://www.w3schools.com/xml/xsl_intro.asp > (10.06.2020). 
Tönen $d, g$ und $h$ sollen Akkorde gebildet werden. Über $d$ ist das $g$ die 5 . Terz, bekommt also die Ziffer 5. $H$ ist die folgende Terz, bekommt also die Nummer 6. Die höchste Nummer ist also 6. Über $g$ ist das $h$ die 1. Terz, $d$ ist die 2. Terz, bekommt also die Nummer 2. Über $h$ ist $d$ die 1 . und $g$ die 6 . Terz, also zählt 6 . So zeigt sich, dass der Akkord ein einfacher Dreiklang über $g$ ist, da die Nummer 2 die niedrigste aller höchsten Terzen ist.

Die automatische Harmonieanalyse zielt auf eine einfache Stufenanalyse ohne Angaben der genauen Intervalle zwischen den einzelnen Tönen. ${ }^{9}$ In der oben beschriebenen Grundtonbestimmung wird lediglich der Grundton berechnet, bei dem es die niedrigsten "Kosten", also die dichteste Terzschichtung, gibt. Nun ist nicht jedes Stück in einem homophonen Satz geschrieben, nicht jeder Ton in einem ermittelten Klang ist harmonierelevant. Es gibt Durchgangstöne, Wechseltöne und Vorhalte, die die Berechnung der Grundtöne verfälschen können. Deshalb werden in einem weiteren Schritt solche harmoniefremden Töne herausgefiltert. Für die Einordnung der harmoniefremden Töne werden an jede Note im Vorfeld Informationen zu ihren nachfolgenden bzw. vorangegangenen Noten und den jeweiligen Intervallen hinzugefügt. Die harmoniefremden Töne haben eine satztechnische Funktion und unterliegen bestimmten Regeln. Alle Töne, die eine Quarte, Sexte, Septime, None oder Sekunde über dem aktuell errechneten Grundton liegen, werden untersucht. Dank der Formalisierung der Noten mit MEI und relativ strikten Notensatzregeln können so Algorithmen formuliert werden, die auf die Daten angewendet werden. Beispielsweise muss bei einem Vorhalt die "vorgehaltene", dissonante Note auf einer betonten, schweren Zählzeit liegen und sie muss sich nach unten in den entsprechenden Akkordton, zum Beispiel von einer Quarte in die Terz, also schrittweise, auflösen. Wenn solche Bedingungen auf Noten zutreffen, werden diese Töne entweder aus der Akkordbewertung herausgerechnet und in der Anzeige ausgegraut (Durchgangs- oder Wechselnoten) oder in ihrer aufgelösten Form bewertet (Vorhalte). Dadurch wird der Akkord bzw. der Grundton beeinflusst und in einem zweiten Durchgang der Terzschichtung (s.o.) neu interpretiert.

Im dritten Schritt werden die ermittelten Akkorde mit der vorherrschenden Tonart in die jeweiligen Stufen umgerechnet. Steht beispielsweise ein Akkord auf dem Grundton $g$ und liegt in einem Fenster, das in der Tonart G-Dur bzw. g-Moll steht, wird die Stufe "I" herausgeschrieben (s. Abb. 3, T. 1). Zudem können aus der oben beschriebenen Grundtonerkennung Septim- und Nonerweiterungen übernommen werden, ebenso wie Umkehrungen, die mit den jeweiligen Ziffern an den Stufensymbolen sichtbar sind.

Es kann allerdings vorkommen, dass bei der Berechnung der Grundtöne bzw. Akkorde mehrere Interpretationen mit den gleichen Kosten entstehen. Aufgrund des beschränkten Platzes im

9 Eine genaue Akkordbestimmung, die unabhängig von einer bestimmten Tonart ist, wurde für die VideApp-Arr entwickelt. Die App entstand im Projekt Beethovens Werkstatt und vergleicht MEI-codierte Werke Beethovens mit von ihm angefertigten Bearbeitungen dieser Werke. Eine Perspektive dieses Vergleichs ist ein Harmonievergleich, bei dem Akkorde analysiert und mit Akkordsymbolen versehen werden. So können eventuelle harmonische Abweichungen zwischen den Fassungen erkannt werden, vgl. <https://videapp-arr.beethovens-werkstatt. de und https://beethovens-werkstatt.de/glossary/harmonievergleich/> (12.06.2020). 
Rendering kann nur eine Interpretation angezeigt werden. Eine Entscheidung für die "richtige" Interpretation des Akkords kann aufgrund von Kadenzabfolgen am besten mit den Stufen erfolgen. Somit wurde die Regel implementiert, dass diejenige Interpretation bevorzugt wird, die auf der gleichen Stufe wie ihr Vorgänger-Akkord steht. Wenn diese Regel nicht angewendet werden kann, werden die Interpretationen nach Kadenzabfolgen bevorzugt. Für diese Analyse wurden die einfachen Regeln I-IV, I-V, V-I, IV-I und II-V implementiert, mit denen eine Priorisierung der Interpretationen erfolgen soll.

In der Analyse-Ansicht des Studienbuch-Viewers (Abb. 3) ist die Übertragung der Noten nun nicht mehr seitenbasiert, sondern werkbasiert, was bedeutet, dass eine Auswahl der in dem Studienbuch befindlichen Stücke in einem drop-down Menü möglich ist. Die Seitenaufteilung wurde hier nicht beibehalten und die o. g. verbalen Wiederholungsanweisungen wurden als Noten ergänzt, sodass ein vollständiger Werktext zu sehen ist, der damit auch die Grundlage einer Analyse bieten kann. In der Analyse-Ansicht kann ebenfalls das Profil der KrumhanslSchmuckler-Tonarterkennung ausgewählt werden. Die Profile haben unterschiedliche Gewichtungen der Töne, die für eine Tonart relevant sind. Zudem kann die Fensterbreite der Tonarterkennung eingestellt werden: von maximal, $d$. h. das ganze Stück betreffend, womit nur eine Tonart berechnet wird, bis zu taktweise, wo evtl. in jedem Takt eine neue Tonart berechnet wird. In Abb. 3 wurde die Fensterbreite von acht Takten gewählt. Man kann sehen, dass drei unterschiedliche Tonarten in dem ganzen Stück berechnet wurden: G-Dur (blau), D-Dur (rot) und C-Dur (grau) und sich die Stufen je nach zugrundeliegender Tonart unterscheiden. Im dritten Abschnitt des Stücks gibt es eine Tonartänderung, die durch ein Auflösungszeichen erkennbar ist. Für den Krumhansl-Schmuckler-Algorithmus ist dieses Zeichen jedoch nicht von Bedeutung. Durch die veränderte Verteilung der Tonhöhen erkennt er aber, dass in diesem Bereich jetzt die Tonart C-Dur bis zum Ende des Stücks vorherrscht.

Ziel dieses Projekts und der Konzipierung einer halb-automatischen Harmonieanalyse war nicht, die bestehenden Programme zu verbessern oder ein viel besseres und genaueres Analyse-Werkzeug entwerfen zu wollen. Vielmehr sollten die bestehenden Algorithmen aus der MIR in einer benutzerfreundlichen, „kommandozeilenlosen“ Umgebung nachgenutzt, mit musikwissenschaftlichen bzw. musiktheoretischen Herangehensweisen kombiniert und die Grenzen ihrer Anwendbarkeit auf MEI-Daten erprobt werden. Dabei zeigte sich, dass der KrumhanslSchmuckler Algorithmus sich bei großen Fensterbreiten, z.B. auf ein ganzes Stück angewendet, als sehr treffsicher in der Bestimmung der Tonarten erwies. Bei kleineren Fensterbreiten und somit weniger Daten (z.B. einzelnen Takten) sind die Ergebnisse unsicherer und teilweise auch fehlerhaft. Dennoch konnte gezeigt werden, dass Tonartänderungen (mit der richtigen Einstellung) durchaus sichtbar werden. Die Implementierung der Terzschichtung zur Akkorderkennung, das Herausfiltern der akkordfremden Töne sowie die Regeln der Kadenzabfolge sind andererseits musiktheoretische Regeln, die in XSLT formalisiert wurden. Hier zeigte sich, dass erweiterte oder je nach musikalischem Kontext variierende harmonische Besonderheiten in 
den Werken mit den sehr allgemein gehaltenen Regeln der Skripte nicht erfasst werden konnten. Da die Analyse immer von einem Grundton ausgeht, können beispielsweise auch keine verkürzt notierten Akkorde erkannt werden, wodurch die Ergebnisse teilweise verzerrt werden. Bei Stücken, die in grundlegenden Akkorden und einfachem Tonsatz gehalten sind, sind die Algorithmen und Regeln jedoch meist problemlos anwendbar und geben passable Ergebnisse aus. Die hier konzipierte Analyse bietet noch viel Luft und Spielraum nach oben und z. B. die Akkorderkennung wurde bereits im Projekt Beethovens Werkstatt in der erwähnten Vide-App aufgegriffen und verbessert.

Zitation: Agnes Seipelt, „Digitale Edition und Harmonische Analyse mit MEI von Anton Bruckners Studienbuch", in: Brückenschläge zwischen Musikwissenschaft und Informatik. Theoretische und praktische Aspekte der Kooperation, in Verbindung mit der Fachgruppe Digitale Musikwissenschaft hrsg. von Stefanie Acquavella-Rauch, Andreas Münzmay und Joachim Veit (= Musikwissenschaft: Aktuelle Perspektiven. Bericht über die Jahrestagung der Gesellschaft für Musikforschung 2019 in Paderborn und Detmold, Bd. 3), Detmold, Musikwissenschaftliches Seminar der Universität Paderborn und der Hochschule für Musik Detmold, 2020, S. 105-113, DOI: 10.25366/2020.100 


\section{Abstract}

The poster shows the results obtained in the project "Digital Music Analysis with the Techniques of the Music Encoding Initiative (MEI) using Anton Bruckner's compositional studies as an example" (2017 to 2019). On the one hand, the project had the goal of presenting a digital edition of Anton Bruckner's study book, which he produced during his lessons with Otto Kitzler from 1861 to 1863. An edition of the music in the textbook encoded with MEI and displayed using Verovio and the facsimile can be displayed simultaneously. On the other hand, an automated harmonic analysis of this music was to be designed. For this purpose, keys are recognized using the Krumhansl-Schmuckler algorithm that is based on a resource of pitch classes which are compared with reference values and thus their similarity is calculated. Based on this, chord recognitions are carried out, which are then linked to the keys in the last step and converted to a roman numeral analysis.

\section{Kurzvita}

Agnes Seipelt, geb. 1989 in Peine, studierte von 2009 bis 2014 Musikwissenschaft und Französisch im Zwei-Fach Bachelor sowie von 2014 bis 2017 Musikwissenschaft mit Profilierungsbereich "Digitale Edition“ im Master an der Universität Paderborn und dem Musikwissenschaftlichen Seminar Detmold/Paderborn. Von 2017 bis 2019 war sie Mitarbeiterin im Forschungsprojekt „Digitale Musikanalyse mit den Techniken der Music Encoding Initiative (MEI) am Beispiel der Kompositionsstudien Anton Bruckners" der Österreichischen Akademie der Wissenschaften. Aktuell ist sie wissenschaftliche Mitarbeiterin im Forschungsprojekt "Beethovens Werkstatt" und promoviert zum Thema Modellierung von Schreibprozessen. 


\section{Digitale Edition und Harmonische Analyse mit MEl von Anton Bruckners Studienbuch}

$>$ Kooperationsprojekt der Österreichischen Akademie der Wissenschaften (ÖAW) mit dem Zentrum Musik | Edition | Medien (ZenMEM)

$>$ Laufzeit: 2017-2019

$>$ Leitung: Dr. Robert Klugseder

> Mitarbeiter*innen: Paul Gulewycz, Agnes Seipelt Peter Provaznik

$>$ Beratend: Dr. Johannes Kepper, Laurent Pugin

$>$ Förderung: GO!DIGITAL 2.0

\section{Studienbuch \& Digitale Edition}

- Studienbuch von Anton Bruckner, das er während seiner Kompositionsausbildung bei Otto Kitzler von 1861-1863 anlegte $^{1}$

- 326 Seiten: Übungen zu Schlussbildungen und Modulationen, Formenlehre und seine sog. „Studiensymphonie“

- Kodierung des musikalischen Texts mit MEI ${ }^{2}$

- XML-basierte Auszeichnungssprache für Musik; maschinenles- und prozessierbar

- Kodierung von editorischen Phänomene wie Varianten und Herausgeberzusätzen

- Kodierung von Phänomenen wie Streichungen $(<\mathrm{del} />)$ und Hinzufügungen $(<\mathrm{add} />$ ); farblich markiert und umschaltbar

- Rendering des Notentextes mit Verovio ${ }^{3}$
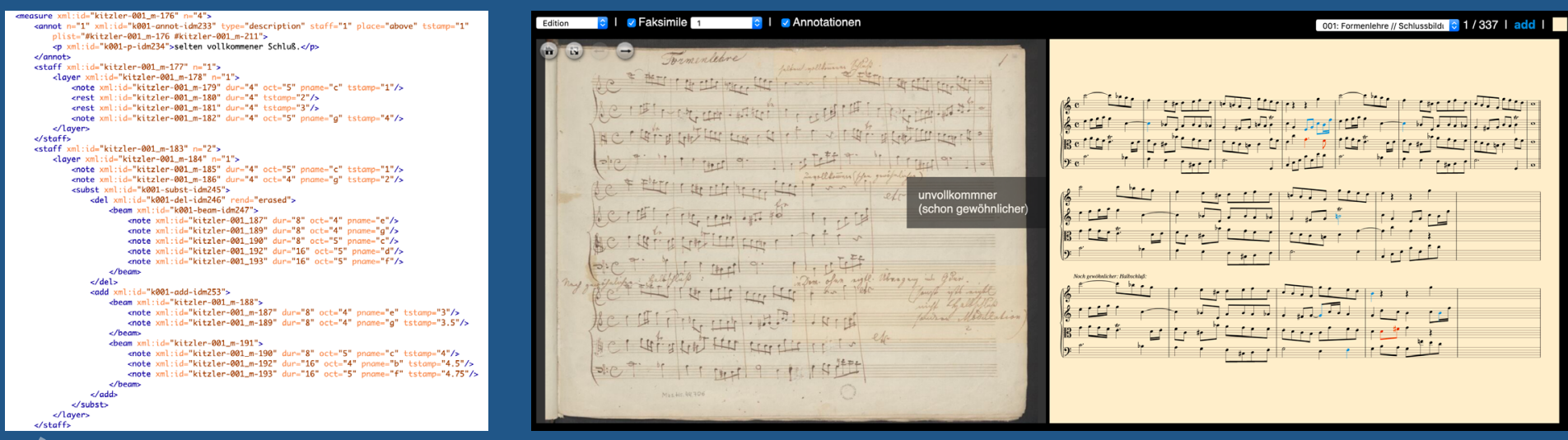

Abb. 2: Anton Bruckner: Kitzler-Studienbuch, S. 1, Digitalisat mit Transkription, https://apps-

\section{Harmonische Analyse}

\section{Tonartenerkennung}

- Krumhansl-Schmuckler-Algorithmus

- "Auszählen" der unterschiedlichen klingenden Töne in einem bestimmten Abschnitt

$\rightarrow$ Input-Vektor (x) der Tonhöhenverteilung: z.B. 12, 1, 6, 5.5, 0, 34, 0, 16.5, 0, 15, 9,0 (12x C, $1 \times$ Cis/Des, $6 \times$ D...)

$\rightarrow$ Abgleich mit Referenzwerten $(y)$ aus Hörexperimenten und Korpora (PiersonKorrelation):

$$
R(x, y)=\frac{\sum\left(x_{n}-\bar{x}\right)\left(y_{n}-\bar{y}\right)}{\sqrt{\sum\left(x_{n}-\bar{x}\right)^{2} \sum\left(y_{n}-\bar{y}\right)^{2}}}
$$

- Referenzwerte zeigen die Verteilung von Tonhöhen in jeder Tonart an (wie gut passt $\mathrm{C}$ in die G-Dur Tonleiter?)

z.B. $6.35,2.23,3.48,2.33,4.38,4.09,2.52,5.19,2.39,3.66,2.29,2.88$ für C-Dur

- Durch Abgleich erhält man einen Index $(R)$, der angibt, wie gut der Input-Vektor zu jeder Tonart passt

$\rightarrow$ der höchste Wert wird als die wahrscheinlichste Tonart angenommen

- Einstellung der Fensterbreite (taktweise) möglich, Berechnung mit

überlappendenden Bereichen

$\rightarrow$ mehrere Tonarten für einen Bereich möglich

$\rightarrow$ Tonartänderungen werden sichtbar

- Darstellung der Tonarten mit farbigen Balken

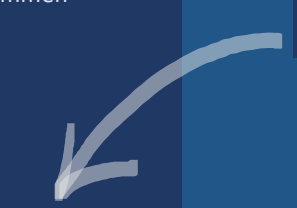

Stufenanalyse

- Kombination der aktuellen Tonart mit konkreten Akkorden

- Anzeige der Stellung und Zusatztöne (Septakkorde)

- Bei mehreren Akkordinterpretationen des Algorithmus: Es wird entweder die Stufe angezeigt, die vorher schon erklingt (1) oder es wird nach Kadenz-Abfolgen entschieden (2):

(1) $\mathrm{V}-\mathrm{h} / \mathrm{V}$

(2) $\mathrm{I}-\mathrm{IV}-\mathrm{IH} / \mathrm{V}$

\section{Akkorderkennung}

- Vertikale Untersuchung von mind. 2 unterschiedlichen Tonhöhen

$\rightarrow$ Berechnung einer möglichst dichten Terzschichtung zur Grundtonerkennung
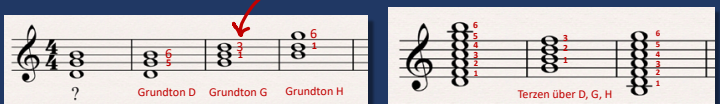

- Mehrere Interpretationen möglich; werden in die Daten geschrieben

- „Herausfiltern" von akkordfremden Tönen:

- Alle Töne, die nicht 3, 5, 7 (9) vom Grundton sind, sind harmonisch irrelevant und sollen aus der Akkordbetrachtung herausgerechnet werden

- Erkennung von Durchgängen, Wechseltönen und Vorhalten mit Algorithmen

- z.B. Durchgang: muss auf unbetonter Zählzeit liegen, vorherige und

nachfolgende Noten liegen Sekunden über und unter der betreffenden Note

- Vorhalte: erklingender Vorhalt wird nicht in die Harmonie gerechnet, sondern die Auflösung

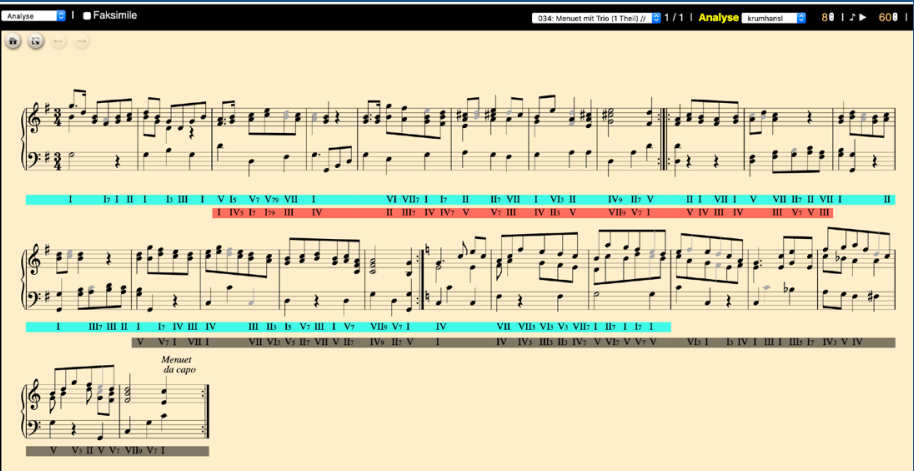




\section{Brückenschläge zwischen Musikwissenschaft und Informatik}

Theoretische und praktische Aspekte der Kooperation

Herausgegeben von Stefanie Acquavella-Rauch,

Andreas Münzmay und Joachim Veit 
Brückenschläge zwischen Musikwissenschaft und Informatik 


\section{Musikwissenschaft: Aktuelle Perspektiven}

Bericht über die Jahrestagung der Gesellschaft für Musikforschung 2019 in Paderborn und Detmold

Herausgegeben von Rebecca Grotjahn und Nina Jaeschke

Band 3 


\section{Brückenschläge zwischen Musikwissenschaft und Informatik}

Theoretische und praktische Aspekte der Kooperation

Beiträge der Symposien zur Digitalen Musikwissenschaft

Osnabrück 2018 und Paderborn 2019

im Rahmen der Jahrestagungen der Gesellschaft für Musikforschung

In Verbindung mit der Fachgruppe Digitale Musikwissenschaft herausgegeben von

Stefanie Acquavella-Rauch, Andreas Münzmay und Joachim Veit

Detmold: Musikwissenschaftliches Seminar der Universität Paderborn und der Hochschule für Musik Detmold 2020 
DOI: $10.25366 / 2020.87$

Online-Version verfügbar unter der Lizenz: Urheberrecht 1.0, $<$ https://rightsstatements.org/page/InC/1.0/?language =de >

Bibliografische Information der Deutschen Nationalbibliothek

Die Deutsche Nationalbibliothek verzeichnet diese Publikation in der Deutschen Nationalbibliografie; detaillierte bibliografische Daten sind im Internet über http://dnb.d-nb.de abrufbar.

\section{Impressum}

Redaktion: Stefanie Acquavella-Rauch, Andreas Münzmay und Joachim Veit Satz: Nina Jaeschke und Joachim Veit

(C) Musikwissenschaftliches Seminar der Universität Paderborn und der Hochschule für Musik Detmold 2020 


\section{INHALT}

Rebecca Grotjahn, Nina Jaeschke

Vorwort zu Band 1-3

IX

Stefanie Acquavella-Rauch, Andreas Münzmay, Joachim Veit

Brückenschläge zwischen Musikwissenschaft und Informatik - Vorbemerkung

$\mathrm{XI}$

\section{KOLLABORATIONEN - KO-LABORATORIEN}

\section{Reinhard Keil}

Der Computer als Denkzeug für hermeneutische Arbeit

\section{Ulrich Konrad}

Philologie und Digitalität. Perspektiven für die Musikwissenschaft im Kontext fächerübergreifender Institutionen

\section{Gudrun Oevel}

Infrastruktureinrichtungen in Forschungsprojekten - Spagat oder Chance?

\section{Dennis Ried}

Erhebung, Transformation und Präsentation digitaler Forschungsdaten

Anna Neovesky, Frederic von Vlahovits

IncipitSearch - Leitfaden zur Zusammenarbeit

Elisabeth Treydte

Clara Schumann \#digital. 40 Jahre Archiv Frau und Musik und der Start in die Digitalisierung

\section{TEXT/DATEN/PROZESSE}

\section{Christine Siegert}

Komponisten-Gesamtausgaben im digitalen Zeitalter: Perspektiven

und Reflexionen am Beispiel Ludwig van Beethovens

Markus Neuwirth, Johannes Hentschel, Martin Rohrmeier

Perspectives of Musical Corpus Studies: The Annotated Mozart Sonatas

Agnes Amminger, Franz Kelnreiter

Leopold Mozarts „Gründliche Violinschule". Zur Textcodierung und -präsentation einer digitalen Edition

\section{Oleksii Sapov}

Algorithmische Automatisierung komplexer Notationsregeln in MEI-XML am Beispiel von Versetzungszeichen 


\section{Susanne Cox, Richard Sänger}

Digitale Fassungsvergleiche am Beispiel von Beethovens Eigenbearbeitungen

Agnes Seipelt

Digitale Edition und Harmonische Analyse mit MEI von Anton Bruckners

Studienbuch

Stefanie Acquavella-Rauch

Musikalische Schaffensprozesse 2.0 - Inkorporation audiovisueller Medien

der populären Musik in Methoden der digitalen Edition

\section{DIGITAL(ISIERT)E MATERIALITÄTEN}

\section{Miriam Akkermann}

(Musik)Instrument (im) Computer

\section{Daniel Fütterer}

Herausforderungen bei der Kodierung von Paratext am Beispiel Neuer Musik mit Live-Elektronik

\section{Matthias Pasdzierny}

How much is the glitch? Das digitale Paradigma als Herausforderung

und Chance für die historische Musikwissenschaft

\section{Shintaro Miyazaki}

Musik für Maschinen?! - Wo sich die Wissenschaft der Medien, des Computers und der Musik treffen und wie sie zusammenarbeiten könnten

\section{MUSIKGESCHICHTE(N) IM NETZ}

\section{Matthias Tischer}

Musikgeschichte der DDR: Ein Pilotprojekt zur digitalen Musikvermittlung

Annette van Dyck-Hemming, Jan Eberhardt, Melanie Wald-Fuhrmann

Ansätze zur Analyse historischer Netzwerke mit Neo4j® - Aus der Projekt-Werkstatt der Datenbank zur Fachgeschichte der Musikwissenschaft

Axel Beer, Martin Bierwisch, Kristina Krämer

Das MMM2 - Ein regionalgeschichtliches Onlinelexikon der Arbeitsgemeinschaft für mittelrheinische Musikgeschichte

\section{Matej Santi}

Was erzählt Fritz Kreislers Geige?

\section{Elias Berner}

Alle Menschen werden Brüder?! Ein historisches Dokument aus dem

Nationalsozialismus in den sozialen Medien 
Gabriele Buschmeier in memoriam 